\title{
UNASUR: construcción de una diplomacia regional en materia de salud a través de políticas sociales
}

\author{
UNASUR: building a regional health diplomacy with \\ social policies
}

Pia Riggirozzi ${ }^{*}$

\section{Resumen}

La lucha contra la pobreza y el acceso a mejores condiciones de vida, incluido el reconocimiento de derechos, son desafíos persistentes que afectan de manera desproporcionada a los países en desarrollo y sus poblaciones más vulnerables. Al mismo tiempo, la integración regional en América Latina hoy representa un conglomerado de proyectos que no solo aborda temas comerciales, sino también políticos y sociales, articulados alrededor de nuevos objetivos y prácticas, basados en solidaridad y autonomía. Esto se ha manifestado particularmente en el área de salud, donde -bajo la coordinación de UNASUR-, una innovadora estructura institucional lidera programas, recursos y políticas para mejorar el acceso a la salud en la región y, mediante una nueva "diplomacia regional", la posición frente a actores internacionales en materia de acceso a medicamentos y derecho a la salud. Este artículo explora UNASUR Salud en la región y frente a actores externos, y argumenta que la región debe ser considerada como "espacio para la práctica política” tanto como "actor internacional”, redefiniendo a la vez qué es región y para qué sirve, así como las sinergias entre regionalismo y desarrollo social.

PAlabras Clave: regionalismo - política social regional - diplomacia regional en materia de salud - UNASUR.

Profesora adjunta de Política Internacional, Universidad de Southampton, Reino Unido. P.Riggirozzi@Soton.ac.uk. Recibido el 15 de septiembre de 2014; aceptado el 25 de abril de 2015. Este trabajo se llevó a cabo con el apoyo del Economic and Social Research Council (ESRC), Ref. de subvención ES/L005336/1, y no refleja necesariamente las opiniones del ESRC. 


\section{Abstract}

Poverty reduction and access to better conditions of wellbeing, including recognition of rights, are persisting challenges in developing countries and their populations. In recent years, the terms of regionalism in South America have been redefined to embrace new objectives and practices beyond economic integration. Since the inception of the Union of South American Nations (UNASUR), health became a 'locus for integration', creating a new framework for formal integration in this policy area. The significance of regional health governance has, therefore, to be seen in the process of region-building but also as new practices projected externally through 'regional health diplomacy'. From this perspective, the region can be considered both as a 'space for policy practice' and as an 'actor'. This paper explores UNASUR's health diplomacy policies and politics within the region and vis-à-vis external actors in relation to access to medicine and rights to health. It is argued that new policies and the negotiation of regional health policies are redefining what region is and is for, and the synergies between regionalism and social development.

KEYWORDS: regionalism - regional social policy - regional health diplomacy - UNASUR. 
PiA Riggirozzi

UNASUR: construcción de una diplomacia regional en materia de salud a través de políticas sociales

\section{INTRODUCCIÓN}

Desde hace ya más de dos décadas, la discusión político-académica se ha centrado en las consecuencias sociales de la globalización económica y modalidades de reducción de la pobreza, mediante campañas globales y transfronterizas, para establecer un piso de protección social, formas de transferencia y financiación para el desarrollo. Sin embargo, los esfuerzos por construir prácticas transfronterizas de inclusión social, incluso en Europa, y particularmente en América Latina en los años ochenta y noventa, usualmente apuntaron a eliminar las barreras al comercio y fueron socavados por la continua reducción de la participación del Estado en la inversión social (Franco y De Filippo, 1999; Grugel, 2005). Consecuentemente, el campo de investigación sobre integración y regionalismo ha tendido a enfatizar dinámicas de comercio y seguridad, relegando la discusión sobre el lugar que ocupan otros proyectos políticos y sociales en el proceso de construcción regional. Este ha sido particularmente el caso en el estudio y la práctica del regionalismo en las Américas, donde el desacople entre integración económica y políticas sociales ha sido consecuencia inexorable del camino seguido por el proyecto neoliberal.

Sin embargo, las crisis abren oportunidades para revertir el orden y reacomodar estrategias y visiones. $\mathrm{Al}$ aflojar el arnés del neoliberalismo como principio organizador de la economía política en América Latina, desde principios del corriente siglo se pudo dar cauce a un proceso de transformación y recomposición de las políticas nacionales y regionales (Grugel y Riggirozzi, 2012). De hecho, es posible argumentar que en un contexto definido por un fuerte cuestionamiento de las políticas de desregulación económica y financiera a raíz de las crisis financieras en América Latina, a principios de este siglo y, según se prevé, en los países centrales hacia el final del mismo, la adopción de enfoques mixtos y generalmente pragmáticos debería desempeñar una papel más dinámico para el Estado y para las instituciones regionales en pos de una agenda social y de desarrollo humano o, como decía Scharpf (1996), de "integración positiva".

En este contexto, nuevas instituciones, fuentes de financiamiento y prácticas al amparo y liderazgo de proyectos regionalistas como la Unión de Naciones Suramericanas (UNASUR), están reformulando los contornos de la política regional, más allá de los tradicionales objetivos de mercado, alrededor de principios de solidaridad y autonomía regional. Puede argumentarse que la nueva configuración político-institucional del regionalismo sudamericano responde a un nuevo consenso regional, que sostiene que las condiciones más apropiadas para afrontar muchos de los actuales problemas sociales se consiguen a escala regional. En este marco, y como analiza este artículo, el área de la salud 
es un ejemplo paradigmático que persigue ampliar la cooperación regional en materia de políticas públicas, (re) distribuir recursos materiales y humanos, y consolidar posiciones comunes en las negociaciones en foros internacionales, para mejorar el acceso a medicamentos y el derecho a la salud.

En este escenario, los términos de la integración regional en América del Sur demandan una nueva mirada crítica y de reflexión política, para entender las oportunidades que ofrece la UNASUR como agente redistributivo en la región y como actor en el contexto de la diplomacia global. El presente trabajo aporta a esta reflexión, indagando sobre la capacidad de las organizaciones regionales de establecer parámetros de acción y cooperación para el diseño, implementación y negociación de políticas que favorezcan el desarrollo social en los países miembros. Al mismo tiempo, se argumenta que la acción conjunta no solo debe evaluarse dentro del espacio regional, sino también a la luz de la capacidad de la región de actuar conjuntamente en foros de gobernanza internacional. Para entender estas dinámicas regionales no es suficiente apelar a las explicaciones dominantes centradas en relaciones causales entre dependencia del comercio internacional, presiones de mercado y opciones de política en los países en desarrollo. Tampoco son suficientes los supuestos habituales sobre el éxito de la integración, medido en función del comercio intrarregional y de la supranacionali- dad. Necesitamos, en cambio, explorar "juegos ignorados" y actores que actualmente están redefiniendo la región como escenario de interacción política y como actor internacional en áreas específicas.

El análisis procede en cuatro partes. En la primera se examinan la construcción regional y el regionalismo en el marco de los cambios político-económicos ocurridos en América Latina. En la segunda se define el regionalismo social a la luz de la experiencia latinoamericana. En la tercera parte se evalúan procesos que facilitan la (re)conexión entre el regionalismo y la política social en América Latina, concentrando el análisis en instituciones, movilización de recursos y prácticas del Consejo de Salud Suramericano de la UNASUR. Finalmente, la cuarta sección ofrece reflexiones sobre la finalidad social del regionalismo y sus desafíos. El artículo concluye con una discusión sobre la medida en que realmente se puede hablar de un giro social del regionalismo.

\section{REGIÓN Y REGIONALISMO EN CONTEXTOS DE CAMBIO}

Más allá de la apelación geográfica, el concepto región puede entenderse como un lugar de deliberación y acción colectiva en ámbitos políticos determinados. En efecto, puede definirse como un campo convencional de instituciones y regulaciones (una esfera de representación territorial) o 
como un ámbito de sociabilidad más informal (un espacio para la acción), o bien ambos. Diversas regiones, consiguientemente, están formadas y operan de variadas maneras y son desarrolladas por diversas formas de interacción social y política, lideradas por actores que comparten motivaciones, preocupaciones, ideologías, metas y percepciones acerca de qué es región y para qué sirve. La construcción regional, en pocas palabras, es la expresión de dichas acciones, así como el espacio en que ellas se desarrollan (Hurrell, 1995). Desde el punto de vista institucional, como toda forma de gobernanza, el regionalismo es una fórmula de coordinación entre diversos actores y proyectos políticos. En sentido amplio, el regionalismo puede seguir una variedad de trayectorias y adoptar diversos ritmos; inclusive, al interior de una misma región pueden manifestarse proyectos superpuestos o en competencia (Briceño Ruiz, 2010; Sanahuja, 2012). Asimismo, puede organizarse y expresarse a través de variadas formas institucionales, abriendo -a su vez- oportunidades para la participación política y la proyección de políticas dentro y fuera del espacio geográfico (Telò, 2001; Söderbaum y otros, 2005; Bretherton y Vogler, 2006).

Para los académicos del llamado enfoque del Nuevo Regionalismo, la construcción del regionalismo denota dos dinámicas fundamentales: por un lado, un sentido de identidad y pertenencia por parte de actores estatales y no estatales, a partir de valores, normas e instituciones compartidas, que gobiernan su interacción dentro de un área geográfica y política, lo que se entiende como autoreconocimiento. En el Nuevo Regionalismo, la ambición política de establecer una identidad y una coherencia regionales (en una comunidad regional ideal) se entiende como un aspecto fundamental, sobre todo cuando se trata de aspectos no territoriales, de preeminencia política (Hettne, 1999). Por otro lado, el grado de institucionalidad y legitimidad, la capacidad de identificar y formular políticas, y la existencia de instrumentos de política permiten desarrollar capacidad efectiva de negociar con otros actores en el sistema internacional, esto es, reconocimiento externo por parte de otros (Hettne, 1999, 2008; Hettne y Söderbaum, 2000; Telò, 2007).

A pesar de la riqueza de estos argumentos, los estudios que explican región como acción colectiva y como actor internacional, se han centrado casi exclusivamente en el rol de la Unión Europea como punto de partida para el estudio de otras regiones (Van Langenhove y Warleigh-Lack 2011). Esto no se debe a negligencia académica, sino a que en las Américas la construcción regional históricamente se concibió como un cálculo de realpolitik, para equilibrar desafíos externos en un marco de globalización (es decir, de hegemonía de los Estados Unidos, competitividad económica de la Unión Europea, capital internacional 
y globalización neoliberal) y compromisos nacionales de desarrollo y, de manera indirecta, electoralistas.

Motivaciones y cálculos económico-estratégicos han definido la práctica y el estudio del regionalismo en América Latina, de acuerdo con una concepción binaria que contrasta el regionalismo "viejo/cerrado" con el regionalismo "nuevo/abierto", a partir de una óptica de política comercial (Gamble y Payne 1996; Phillips 2003; Tussie 2009). La construcción regional así entendida se concibió como un proyecto dominado por el "poder disciplinador" del mercado y la globalización (Franco y De Filippo, 1999; Alvarez, 2011: 23).

Estas percepciones se volvieron más urgentes a medida que el régimen de comercio se relanzó bajo el paraguas de la Organización Mundial del Comercio (OMC). La creación del MERCOSUR, en 1991, y el Tratado de Libre Comercio de América del Norte (NAFTA), suscrito con Canadá y México en 1994, por ejemplo, son parte de la noción de "regionalismo abierto" (Phillips, 2003: 329). Para Estados Unidos, mientras tanto, este contexto abrió una nueva oportunidad para una ambiciosa agenda definida por la "Iniciativa para las Américas", lanzada en 1990 por el presidente George W. Bush, con la visión de conducir a un Área de Libre Comercio de las Américas (ALCA), con plazo para su firma en 2005. El proyecto del ALCA encarna la ambición más extrema en términos regionalistas, en torno a las relaciones intrahemisféricas bajo el ala de Estados Unidos. Al mismo tiempo, refleja la antigua visión del panamericanismo bajo la égida del neoliberalismo. Sin embargo, el establecimiento del ALCA como parangón del regionalismo encabezado por Estados Unidos fue un arma de doble filo. Como sostiene Tussie (2009: 178), a pesar de que el ALCA ha provocado reacciones de pánico en una serie de países que temieron quedar excluidos, la idea del regionalismo neoliberal fue muy controvertida desde el principio y combatida por reacciones de resistencia que constituyeron factores adversos para lograr en la región una cohesión social, capaz de sostener y legitimar la profundización de políticas neoliberales a nivel nacional y regional. En efecto, más allá del control de la inflación, que en países como Argentina significó un freno real a una situación de caos socioeconómico que casi puso en jaque la continuidad democrática, el proyecto neoliberal fracasó fundamentalmente en sus promesas sociales, afectando duramente las condiciones de pobreza y desigualdad en los países de la región (Szekely y Birdsall, 2003). No sorprende que para finales de la década del noventa, en América Latina el neoliberalismo fue perdiendo fuerza. La tasa de crecimiento económico de la región durante estos años noventa, inferior a las cifras de crecimiento promedio registradas entre los años sesenta y setenta, fue altamente decepcionante (op cit.). La desaceleración del crecimiento provocada por 
las dificultades en materia de divisas, el aumento de la deuda (especialmente pronunciado en Argentina) y la creciente conciencia de que la situación social -legada del modelo neoliberalera insostenible, fue el contexto en que se manifestó un cambio de actitud hacia las reformas pro-mercado. Crecientemente, el programa del regionalismo "abierto", estrechamente ligado con la economía neoliberal y el liderazgo de Estados Unidos, fue perdiendo terreno y dinamismo tanto en su carácter ideológico-político como en lo económico.

La percepción de que el neoliberalismo ha fracasado como proyecto capaz de proveer crecimiento económico y democracia social, abrió un espacio de debate y búsqueda de vías alternativas y autónomas para el desarrollo. En este caso, tanto el Estado como la región se convirtieron en actores esenciales en la redefinición de las reglas de juego. La creciente retórica nacionalista que tuvo lugar a través de América Latina, en particular en el Cono Sur, reenmarcó los términos del debate y la adopción de modelos alternativos de gobernabilidad (MacDonald y Ruckert, 2009).

En medio de una reacción generalizada contra lo que se consideró una excesiva mercantilización de la democracia, nuevas formas de acción estatal permitieron la "recuperación" del Estado y del espacio regional. "Recuperar la región”, en efecto, no solo pasó a ser una manera de resistir el dogmatismo neoliberal, sino como un modo más complejo de acomodar políticas internas y formas de organizar la provisión de bienes públicos. Aunque la idea de una contrahegemonía unificada para reemplazar el neoliberalismo en América Latina es claramente exagerada, desde principios de la década de 2000 la región encauzó proyectos regionales alternativos. Esto se cristalizó, en principio, en la Primera Cumbre de Presidentes de América del Sur del año 2000, cuando la discusión se centró en torno a renovados compromisos sobre principios democráticos y, en un sentido más amplio, del desarrollo para responder a la deuda social frente a las fallas del Consenso de Washington (Sanahuja, 2012). Sin embargo, no fue sino hasta la clausura de las negociaciones del ALCA, en el marco de una nueva Cumbre de las Américas en 2005, que el proceso de integración de América del Sur entró en una nueva fase. La Tercera Cumbre celebrada en Cuzco, Perú, en diciembre de 2004, estableció lo que posteriormente se institucionalizó como la Unión de Naciones Sudamericanas (UNASUR). La Declaración de Cuzco estableció tres objetivos principales: la convergencia entre los acuerdos comerciales dirigidos preexistentes, especialmente el MERCOSUR y la Comunidad Andina; compromisos nuevos para avanzar en la infraestructura física (carreteras, energía y comunicaciones), y la cooperación política en materia de salud y seguridad (Riggirozzi y Tussie, 2012). Ese mismo año, un nuevo proyecto regionalista, liderado 
por Venezuela, también vio nueva luz, la Alianza Bolivariana de las Américas (ALBA).

A pesar de las diferencias ideológicas e institucionales, y más allá del simbolismo político, estos proyectos adoptaron nuevos compromisos a nivel regional, respondiendo a necesidades de desarrollo humano (Sanahuja, 2012). Por supuesto, esto no es simplemente voluntarismo político, sino también una oportunidad dada por la redefinición de las coordenadas de comercio y poder en el escenario internacional, en particular el aumento sin precedentes de la demanda global de productos primarios; los nuevos retos geopolíticos de Estados Unidos, y una renovada oportunidad en la cooperación Sur-Sur y vinculaciones alternativas al comercio y las finanzas. En otras palabras, nuevos catalizadores a nivel nacional e internacional han forjado oportunidades para redefinir objetivos de economía política regional y formas de acción colectiva. En este contexto, el proceso de construcción de región en las Américas demanda una mirada más atenta a los modelos alternativos de desarrollo y política social, de movilización de recursos (materiales y humanos) y de acción colectiva, que reconectan el regionalismo y el desarrollo social.

\section{Regionalismo Y POLÍTICA}

SOCIAL REGIONAL

Tanto las consideraciones sobre el desarrollo social como las formas de maximizar la política social, están ganando protagonismo en el debate sobre el regionalismo, al tiempo que los países tratan de abordar los problemas de pobreza, desempleo y desigualdad social. Mientras la globalización crea riesgos frente a fronteras nacionales cada vez más permeables, la protección social se convierte en un tema fundamental para la gestión y la participación en los procesos de integración política y económica. En particular, desde la década del noventa ha habido un creciente consenso de que, en un mundo cada vez más globalizado, para lograr un desarrollo socialmente equitativo se requieren formas novedosas de pensar y actuar políticamente. Más allá del enfoque sobre políticas sociales como dominio de la política nacional y frente al reconocimiento de una amplia variedad de dominios y espacios de producción, negociación e implementación de cooperación transnacional, puede argumentarse que las causas y soluciones a muchos problemas sociales no se limitan necesariamente a las instituciones, estructuras y marcos nacionales, sino más bien son parte de procesos que se extienden más allá de los límites del Estado-nación (Ortiz, 2007).

Existe una larga trayectoria de cooperación internacional que desde la década del noventa ha proliferado en forma de donaciones, cooperación técnica, financiamiento y ayuda internacional, por medio de organizaciones no gubernamentales internacionales e 
instituciones multilaterales de crédito, en el marco del sistema de Naciones Unidas. Por lo general, se ha partido de la base de que a falta de capacidad o voluntad del Estado, las intervenciones "globales" de organizaciones no gubernamentales privadas y organismos multilaterales de desarrollo, se convierten en mecanismo para la política nacional a través de la redistribución transnacional, regulaciones supranacionales, asesoramiento técnico y cooperación, y ayuda condicionada (Deacon y otros, 2010). En la práctica, los esfuerzos por eliminar la pobreza han sido fundamentales para canalizar transferencias NorteSur y, en algunos casos, definir un "piso" de protección social universal. En América Latina, la política social transnacional, así entendida, adoptó principalmente la forma de políticas compensatorias, es decir, programas de lucha contra la pobreza y la inversión social del Banco Mundial y del Banco Interamericano de Desarrollo, entre otras organizaciones no gubernamentales, para equilibrar el impacto de la apertura comercial y la desregulación del mercado sobre estándares sociales, sobre todo a la luz de la privatización de servicios públicos como la salud, educación y pensiones. Las controversias en torno a estos programas "de política social global", vistos como intervención en respuesta a fallas del mercado o del gobierno, han estado -en general- desvinculadas de un debate más amplio acerca de los modelos de desarrollo y transforma- ción social (Deacon et al., 2010).

Al mismo tiempo, el aumento de los problemas transfronterizos, tales como las pandemias o la inmigración ilegal, desató otra fuente de debate en relación con la necesidad de coordinar mecanismos de vigilancia para el control de las enfermedades transmisibles, así como para mitigar los efectos de una constante transnacionalización y crear mayores oportunidades interfronterizas (Ortiz 2007: 64). En América Latina, por ejemplo, surgieron nuevas formas de cooperación transnacional en el ámbito de la vigilancia de la salud y la migración laboral, así como en áreas específicas consideradas claves para impulsar la competitividad del mercado, como la educación superior y la inversión extranjera (SELA 2010; Deacon et al., 2010). En este marco, la incipiente política social regional puede definirse en torno a su capacidad de asegurar cooperación transfronteriza para la redistribución de los recursos; marcos regulatorios para la convergencia, y armonización de políticas, servicios y, potencialmente, derechos en el ámbito social. Asimismo, la coordinación política en espacios regionales puede ser vista como alternativa a modelos tradicionales de transferencia internacional, tanto en su ayuda al desarrollo como en calidad de recetas de política (Almeida, et al., 2010).

A pesar de este debate, fuera del contexto de la UE hay poca investigación empírica real sobre la política social regional y dinámicas políticas 
relacionadas con sectores específicos. Los estudios comparados sobre regionalismo y políticas regionales, invariablemente se han concentrado en los contornos institucionales de las estructuras regionales o en las políticas de comercio, finanzas y seguridad. Es necesario profundizar nuestra comprensión del lugar de la política social en la construcción regional contemporánea en América Latina, así como sobre tipos de políticas, finalidad, mecanismos y efectos de estas en relación con el desarrollo social. Ello se hace más apremiante de cara a lo que se ha denominado "regionalismo post-neoliberal” en América Latina (Sanahuja, 2012), que ofrece un punto de entrada interesante para revisar la cooperación regional y su sinergia con las políticas sociales.

\section{REGIONALISMO SOCIAL EN América LATINA: UN DESAFío TRUNCADO}

El modo en que se ha desarrollado el regionalismo en las Américas encierra una paradoja: pese a que la construcción regional fue moldeada en el supuesto de que el comercio y la inversión financiera constituyen los principales catalizadores del crecimiento y el desarrollo, algunas "cláusulas sociales" cimentaron la cooperación e implementación de proyectos transfronterizos en la Comunidad Andina de Naciones (CAN) y en el MERCOSUR (Raso Delgue, 2006; Caeta- no, 2007; Draibe 2007; Riesco, 2010). En el caso de la primera, por ejemplo, se crearon dos órganos para administrar desafíos comunes en las áreas de educación y salud. El Acuerdo Hipólito Unanúe y el Convenio Andrés Bello establecieron las bases para la coordinación de las políticas de salud y educación, respectivamente, desde principios de los años setenta. En el área de la salud se implementaron políticas activas relacionadas con la prevención y control de enfermedades que afectan zonas de frontera; en el campo de la educación, por su parte, las políticas se orientaron a la armonización curricular, la movilidad de estudiantes y profesionales, así como programas para asegurar la calidad educativa en la región andina (UNDP, 2011). En lo que respecta al MERCOSUR, la Reunión de Ministros de Salud -en tanto instancia institucional del acuerdofue creada a mediados de los años noventa, a modo de foro para examinar la política de salud y formular estrategias en este campo, como parte de la agenda regional (Decisión CMC $N^{\circ}$ 03/1995). Avances similares se registraron en el área de la educación a partir de la institucionalización del "Sector Educativo del MERCOSUR", en el año 1991, cuyo órgano máximo de decisión es la Reunión de Ministros de Educación, con instancias de coordinación en áreas de trabajo (educación básica, superior y técnica) que dedicaron buena parte de los años noventa a armonizar los indicadores educativos como medida inicial para 
la elaboración de políticas. En efecto, las acciones en materia de educación y de salud impulsaron la política social de la agenda regional, generando actividades de cierto impacto (Perrotta y Vázquez, 2010). Sin embargo, durante los años noventa, los nuevos compromisos y la institucionalización de la cooperación, así como la implementación de políticas sociales, tuvieron una trayectoria errática y de consecuencias variadas para el desarrollo humano en ambos esquemas de integración regional.

En la práctica, estas embrionarias agendas sociales regionales fueron objeto de restricciones presupuestarias, barreras institucionales del propio sistema de integración y dentro de la estructura institucional interna; ausencia de planes de acción, y truncado compromiso político (Franco y De Filippo, 1999; Riesco, 2010). Desde el punto de vista político, estructuras institucionales rudimentarias tanto en la CAN como en el MERCOSUR, a menudo delegaron las decisiones sobre las políticas y la política regional en los procesos intergubernamentales de negociación, entregando la política social a la discreción de los decisores políticos, ministros y actores privados, y a la lucha entre estos por la distribución de los beneficios (Malamud, 2005). En consecuencia, la capacidad de los actores sociales para penetrar el debate político regional y la negociación de las políticas, ha estado severamente recortada por una cultura política regional cerrada a la sociedad, a la par que responde a presiones del mercado.

A pesar de las dificultades, algunas iniciativas han creado oportunidades para el activismo social. Este ha sido el caso del MERCOSUR, a partir del establecimiento del Instituto Social del MERCOSUR (ISM), en 2007, creado para apoyar el trabajo de la Reunión de Ministros y Autoridades de Desarrollo Social del MERCOSUR. El ISM se erige como un eje central para la investigación en materia de política social y la consiguiente recomendación de políticas. De la misma manera, la iniciativa "Somos MERCOSUR" -creada en la presidencia protempore uruguaya del año 2005 e institucionalizada en el bloque en la Cumbre de Córdoba-Argentina, en el año 2006- abrió un nuevo espacio para la formación de una conciencia ciudadana, que permitió establecer un proceso de Cumbres Sociales en paralelo a las Cumbres Presidenciales (desde julio de 2006), generando así un espacio de encuentro entre los gobiernos y la sociedad civil en pos de la discusión y deliberación de prioridades de política y agenda. Mientras que estos hechos e iniciativas institucionales reforzaron una nueva agenda social, la acción política y las normas regionales permanecieron plagadas de ambigüedades procedimentales $y$, fundamentalmente, frenadas por dificultades económicas y financieras, así como por prerrogativas del Consenso de Washington de reducir la inversión estatal en políticas sociales (Di Pietro, 
2003; Grugel, 2005). No resulta sorprendente, por lo tanto, que al inicio de la década los gobiernos de izquierda de la región se pronunciaran en contra del núcleo neoliberal y la tutoría de Estados Unidos, y reorientaran las políticas nacionales buscando sincronizar también políticas de inclusión en escalas múltiples. Esto se vio reflejado en el Tratado Constitutivo de UNASUR, firmado en Brasilia en mayo de 2008, que declara explícitamente los derechos humanos como valor fundamental de la integración, y expresa que es necesario fomentar un proceso integrador en apoyo de la inclusión social y la erradicación de la pobreza. En este marco, también se declara en concreto el "derecho a la salud como la fuerza energética de las personas en el proceso de integración de América del Sur" (UNASUR 2009: 14) .

\section{LA SALUD COMO MOTOR}

REGIONAL

Unas de las notables paradojas que definen la actual economía política de la región son las persistentes disparidades entre el crecimiento económico registrado en la última década y el desarrollo social. A pesar de los niveles de crecimiento sin precedentes y el apoyo a las políticas progresistas en el último decenio, y pese a una fuerte mejora en los índices de pobreza, que entre 1999 y 2011 cayeron más de un 14 por ciento, aún alrededor de 168 millones de personas viven en condiciones de pobreza. Todavía un 30 por ciento de la población subsiste con menos de dos dólares al día, mientras que 66 millones viven en extrema pobreza, percibiendo menos de un dólar al día (CEPAL 2010; 2011: 14). Esta situación se concentra desproporcionadamente en poblaciones económica y socialmente más vulnerables, por lo general localizadas en zonas transfronterizas, por ejemplo entre poblaciones indígenas y rurales pobres, trabajadores inmigrantes, ancianos, mujeres y niños, que también tienden a sobrellevar una carga mayor de enfermedades infecciosas y discapacidades (CEPAL 2011: 9). Muchos estudios reflejan el estrecho vínculo entre enfermedades transmisibles, enfermedades infecciosas y determinantes sociales de la salud, tales como la falta o acceso insuficiente de agua potable, saneamiento, vivienda adecuada, educación y servicios de salud en América Latina (Holveck et al., 2007). Más aún, estas condiciones reducen las oportunidades de aprendizaje, productividad y capacidad de generar ingresos. Algunas cifras alarmantes muestran que en países de ingresos bajos, como Bolivia, Paraguay y Perú, las enfermedades transmisibles ejercen una influencia extrema en términos de calidad y esperanza de vida. En Haití, la incidencia de la tuberculosis es siete veces mayor que en la región, mientras que el dengue y el VIH, aunque un problema considerable y creciente en toda la región, afecta despropor- 
cionadamente a Brasil. La malaria es endémica en 21 países de la región (Barreto et al., 2012). Los profundos niveles de pobreza han sido asociados con la forma en que muchos países de América Latina se han integrado en la economía mundial, agravados por el legado de reformas neoliberales de la década del noventa, que redujo el gasto público en políticas de bienestar social y la participación estatal en las disposiciones de la seguridad sanitaria, de educación y social (Hotez, 2008). Esta sombría situación se ve agravada por los bajos niveles de prestación de servicios sociales y el acceso limitado a medicamentos, obstaculizado por negociaciones comerciales desfavorables con países desarrollados o por acuerdos bilaterales de libre comercio con Estados Unidos y la UE, especialmente con Centroamérica, Chile, Perú y Colombia, que han restringido la flexibilidad para otorgar licencias obligatorias e importaciones paralelas de medicamentos a precios más bajos, situación que afecta especialmente a poblaciones de zonas rurales y tropicales (Echenique Walker, 2012).

En la lucha por el derecho a la salud y el acceso a medicamentos en particular, tanto el MERCOSUR como la Comunidad Andina han tratado de sostener negociaciones colectivas para determinar y reducir los precios en la adquisición de productos farmacéuticos, especialmente en respuesta a la escalada del VIH/SIDA en Brasil, en la década de los noventa (SELA 2008: 56). Pero estas iniciativas han sido más bien ad hoc y se han visto severamente limitadas por las realidades y presiones de economías altamente dependientes de la cooperación internacional y de préstamos condicionados sobre austeridad fiscal, privatización y desregulación de mercados, incluidos el de salud (Almeida, 2010). En este contexto, los países sudamericanos no lograron crear instituciones regionales estables y eficaces para proteger y promover los derechos relacionados con la salud, y crear oportunidades para las personas. Sin embargo, los cambios experimentados por la economía política internacional y nacional en la presente década, han abierto una oportunidad para recrear sinergias intrarregionales y acuerdos regionales para el desarrollo social.

A partir de lo expuesto más arriba, cabe preguntarse por qué la salud se convirtió en motor de la agenda social regional, comparado con otros sectores en que el dinamismo y la visibilidad de UNASUR han sido menores. Mientras que experiencias en el campo de la cooperación en salud en MERCOSUR y la Comunidad Andina se vieron limitadas por necesidades -reales o percibidas- de ajuste estructural y reducción del gasto social en la década del noventa, UNASUR retomó políticas sociales en un espacio político-económico diferente. Asimismo, mientras que los anteriores acuerdos regionales abordaron la salud como respuesta bilateral para hacer frente a determinados problemas transfronterizos relacionados con el control y 
la vigilancia epidemiológica (Buss, 2011), UNASUR recupera la salud no solo como un problema sanitario y de políticas públicas, sino fundamentalmente como uno de derechos y de gobernanza (Buss y Do Carmo Leal, 2009). En otras palabras, UNASUR busca no solo reducir riesgos sanitarios transfronterizos, sino también mejorar la capacidad de representación y negociación en el espacio de la diplomacia global, para ejercer influencia en las políticas de acceso a los medicamentos.

Para lograr estos objetivos, UNASUR desarrolló una innovadora institucionalidad en el área de la salud, estructurada por consejos formados por presidentes, cancilleres y delegados, y por consejos sectoriales, que se ocupan de temas específicos. Mientras que el Secretario General actúa como representante de UNASUR, el órgano superior -en términos decisorios- es el Consejo de Jefas y Jefes de Estado, que establece planes de acción y decide sobre propuestas de los Consejos de Ministras y Ministros de Relaciones Exteriores. Los Consejos Ministeriales constituyen instancias operativas para la implementación de las políticas del organismo y, por ende, representan el componente más dinámico de la estructura de UNASUR. Su creación, organización, definición de objetivos y funciones, tanto como su asignación de tareas a través de diferentes grupos de trabajo, contribuyen a una lógica de especialización del organismo en diferentes áreas de intervención. Una vez aprobado el Tratado Constitutivo, en mayo de 2008, el Consejo de Defensa Suramericano y el Consejo de Salud Suramericano fueron los primeros consejos constituidos para atender estos temas específicos, contando cada uno con dos centros regionales de investigación y promoción de políticas: el Centro de Estudios Estratégicos de la Defensa (CEED) y el Instituto Sudamericano de Gobierno en Salud (ISAGS).

Desde su creación, el Consejo de Salud definió un plan de acción coordinado por Grupos de Trabajo temáticos en cinco programas:

1. Red Suramericana de Vigilancia y Respuesta en Salud.

2. Desarrollo de Sistemas de Salud Universales.

3. Acceso Universal a Medicamentos.

4. Promoción de la Salud y acción sobre los determinantes de la Salud.

5. Desarrollo de Recursos Humanos en Salud (Plan Quinquenal: 2009).

En conjunto con los Grupos de Trabajo, el ISAGS -con sede en Río de Janeiro- actúa como think-tank para la coordinación de la investigación relacionada con estos objetivos, formación de cuadros técnicos, y apoyo en vísperas de negociaciones internacionales, en el marco de la Organización Mundial de la Salud (OMS). Ideológicamente, el ISAGS 
se hace eco del histórico Movimiento Sanitarista brasilero, retomando el principio de la salud como derecho y fundamento de movimientos político-académicos más amplios a favor de la medicina social en América Latina, desde las décadas del sesenta y setenta (Buss y do Carmo Leal, 2009). Políticamente, el ISAGS se nutre del papel internacional de Brasil como actor global en materia de salud, que ha asumido un rol cada vez más protagónico en la última década, en temas de diplomacia global, sobre todo en relación con el acceso a medicamentos y el VIH (Soares de Lima y Hirst, 2009; Ayllón Pino, 2012). En la práctica, el ISAGS actúa como centro de investigación y desarrollo de UNASUR, así como de formación política, de expertos y profesionales para los países miembros. Sus actividades incluyen seminarios, cursos, programas de pasantías en apoyo a reformas de los sistemas de salud y de liderazgo nacional e internacional, y la identificación de capacidad industrial en la región, para coordinar políticas comunes para la producción de medicamentos.

En este contexto, UNASUR opera fomentando la cooperación horizontal o intrarregional, centrada en esta última y ejerciendo diplomacia transversal o extrarregional, buscando redefinir las relaciones Norte-Sur. La cooperación intrarregional refleja nuevos consensos sobre inclusión social y derechos como determinantes sociopolíticos en materia de salud, y se orienta a movilizar y (re)distribuir recursos humanos y financieros para los objetivos planteados en el plan quinquenal. En el caso de intervenciones extrarregionales, esto es sin duda un factor novedoso en relación con el regionalismo del Mercosur o la Comunidad Andina, dado que UNASUR opera como bloque en discusiones en el marco de la Organización Mundial de la Salud y frente a farmacéuticas internacionales.

En la práctica, la acción intrarregional de UNASUR se ha visto en la coordinación de programas de salud y seguridad alimentaria en países como Haití y Chile, que han sufrido catástrofes naturales; en el suministro de ayuda para el saneamiento y la lucha contra el cólera, y en programas de vacunación contra la gripe H1N1 y el dengue (PAHO 2010; UNASUR 2010). Asimismo, el ISAGS, actuando como "capacitador", ha apoyado al Ministerio de Salud de Paraguay y Guyana, para la ejecución de las políticas nacionales relacionadas con la atención primaria y la elaboración de protocolos clínicos, y más recientemente, con reformas hacia la universalización del sector en Colombia, Perú y Bolivia (ISAGS 2013). Otra iniciativa regional liderada por el Grupo Técnico de Recursos Humanos y Gestión de UNASUR ha sido la sistematización y difusión de las mejores prácticas y actividades de capacitación para el apoyo a funcionarios de Ministerios de Salud, Escuelas de Salud pública y negociadores internacionales (Fiocruz 
Agencia de Noticias, 2012). Por último, el ISAGS trabaja en el apoyo a redes de instituciones para implementar proyectos contra enfermedades no transmisibles, como el cáncer y la obesidad, y contra la propagación del $\mathrm{VIH}$, malaria, dengue, tuberculosis, Chagas y otras enfermedades transmisibles, mediante mecanismos de vigilancia, planes de vacunación y medicamentos (UNASUR 2009b).

Para fomentar el acceso a vacunas y medicamentos, en la VII Reunión del Consejo de Salud, en octubre de 2012, los países de UNASUR acordaron establecer un "mapa de las capacidades regionales para la producción de medicamentos", aprobado por el Consejo de Salud, en el cual el ISAGS coordina la identificación de las capacidades industriales existentes en la región, para definir políticas comunes para la producción de medicamentos. Además, se propone elaborar conjuntamente listas de precios de los medicamentos, para luego planificar mecanismos de compras unificadas, a fin de reducir costos. El "Banco de Precios de Medicamentos" constituye una base de datos informatizada con precios que los países de América del Sur pagan por la compra de medicamentos, proporcionando una herramienta de apoyo para la toma de decisiones en la adquisición de estos frente a las farmacéuticas. Estos marcos ayudan a mejorar la información y a generar condiciones para mejorar el acceso a la salud y utilizar de manera más eficiente los recursos públicos.
En lo que respecta a los organismos multilaterales, UNASUR ha estado actuando con una sola voz en la Asamblea Mundial de la Salud, presentando propuestas de reforma para mejorar la representación, gestión y forma (global) en que se definen los problemas de salud y las prioridades en la agenda internacional. UNASUR ha dirigido con éxito discusiones sobre el acceso a medicamentos y el papel de la OMS en la lucha contra la falsificación de productos médicos, que han estado dominadas por la International Medical Products Anti-Counterfeiting Taskforce (IMPACT), organismo liderado por grandes farmacéuticas, Interpol y la Organización para la Cooperación y el Desarrollo Económico (OCDE). A primera vista, el tema de los productos médicos falsificados, de etiquetado engañoso, espurios, de imitación o de calidad subestándar, se remite a la función de fiscalización de la autoridad sanitaria para garantizar seguridad y medicamentos de calidad. No obstante, de acuerdo con lo planteado por UNASUR en la OMS, la falta de precisión y la interpretación de algunos de estos términos significó que muchas veces se utilizaran consideraciones técnicas para restringir la comercialización de productos genéricos, apelando a conflictos relacionados con derechos de propiedad intelectual. UNASUR presentó una posición unificada, pidiendo a los países en desarrollo que tomaran conciencia del riesgo de impedir la expansión de la política de genéricos, tan importante para su- 
perar las barreras de acceso a los medicamentos (Agencia Fiocruz, 2012).

A partir de posiciones emanadas de UNASUR, muchos países se sumaron, planteando que la OMS no podía formar parte de una agencia con actores que ningún interés tenían en la salud pública, sino comercial. Así en la $63^{\mathrm{a}}$ Asamblea Mundial de la Salud se formó un grupo de trabajo que planteó un giro en el enfoque, recuperando la perspectiva de salud pública. En este contexto, UNASUR propuso crear una nueva instancia intergubernamental que reemplazara a IMPACT, para actuar y prevenir la falsificación de productos médicos. Esta resolución fue aprobada posteriormente por la Asamblea Mundial de la Salud en mayo de 2012. Asimismo, UNASUR presionó con éxito para que se iniciaran negociaciones destinadas a llegar a un acuerdo vinculante sobre el apoyo financiero y mejorar las oportunidades de investigación en innovación y financiamiento, para satisfacer las necesidades de los países en desarrollo. Más recientemente, con el liderazgo de Ecuador, UNASUR presentó a debate un plan de acción para un mayor reconocimiento de los derechos de las personas discapacitadas, como parte de un criterio más amplio para abordar los condicionamientos sociales de la salud, que fue aprobado dentro de la normativa de la OMS. ${ }^{1}$

1 Propuesta 'Atención Integral de la Discapacidad' Consejo Ejecutivo de la OMS, 21 a 29 de enero de 2013, Punto 6.5 del orden del día, documento EB 132/10,

\section{EL PROPÓSITO SOCIAL DEL}

\section{REGIONALISMO}

Aún no se conoce, a ciencia cierta, en qué medida las instituciones regionales pueden cerrar la brecha de desarrollo desigual en América del Sur. Sin embargo, la nueva arquitectura institucional, políticas y planes de acción concretos, han abierto un nuevo camino novedoso para la acción colectiva en un campo de alta sensibilidad social, como es la salud, con enormes consecuencias para el desarrollo social, la inclusión y la promoción de derechos.

Los aspectos regulatorios y las prácticas de UNASUR permiten reconectar motivaciones nacionales, modelos de economía política e integración regional, rompiendo con supuestos deterministas sobre la relación regionalismo-globalización.

UNASUR emerge como un sitio para construir consensos, foco para formar cuadros políticos y técnicos, y coordinador industrial, rompiendo con la tradicional transferencia pasiva de conocimientos y tecnologías desde países desarrollados hacia la región. Asimismo, se abren nuevas oportunidades para enfrentar desafíos de la política global.

En segundo lugar, la cooperación regional, en materia de salud, tiene la

archivo en posesión del autor. También, http://upsidedownworld.org/main/ecuador-archives-49/4875-ecuador-pushesfor-greater-south-south-cooperation-andstronger-public-disability-assistance-policies (8/9/2014) 
capacidad para conducir un proceso de transformación, creando nuevos espacios de acción que reconectan modelos de desarrollo y derechos de ciudadanía con la integración regional. Esto se manifiesta no solo en el Plan Quinquenal de UNASUR Salud, sino además en el compromiso internacional de mejorar las condiciones de acceso a medicamentos y de participación de países en desarrollo en los foros internacionales. Más aún, a pesar del largo camino por recorrer en la consolidación de un genuino regionalismo social, la experiencia de UNASUR Salud representa un vehículo y una oportunidad para trazar lecciones que sirvan al desarrollo en otras áreas de política social, como migraciones, medio ambiente, derechos de género, etc.

Por supuesto, este proceso no está exento de contradicciones. Toda política engendra luchas distributivas y de poder que, en el caso de la construcción de la región de América del Sur, pueden definirse por la falta de participación de las organizaciones de la sociedad civil en la definición de las políticas sociales, programas e instituciones de UNASUR. De hecho, la creación de este organismo aún no ha concebido mecanismos nuevos para incorporar a los actores sociales en la definición de las agendas sociales (Serbin, 2012). Se puede argumentar, sin embargo, que instituciones como ISAGS fomentan y generan comunidades epistémicas regionales y comunidades de práctica, trabajando en redes con distintos actores y abriendo nue- vos espacios para la participación de actores no estatales en la definición e implementación de políticas regionales. La presencia de expertos, sanitaristas y académicos coordinando redes temáticas en torno a los objetivos de UNASUR Salud, tiene el potencial de minimizar el exceso de intergubernamentalismo que sustentan los actuales desarrollos regionales. En otras palabras, mientras que en salud el hiperpresidencialismo se tamiza mediante la presencia e influencia de expertos, académicos, técnicos y sanitaristas involucrados en la producción de conocimiento, de políticas y de prácticas transfronterizas, estos espacios y compromisos no reemplazan la falta de un diálogo institucionalizado de carácter más amplio en otras áreas de política regional y, por ende, la capacidad transformadora de UNASUR, como proyecto inclusivo y redistributivo, continúa siendo una historia abierta.

Finalmente, no obstante existen fuertes razones para sostener que las instituciones regionales poseen escasa capacidad ejecutora para garantizar que las declaraciones conjuntas conduzcan a compromisos y estos se traduzcan en la aplicación de políticas, la capacidad de prácticas institucionales como las del ISAGS como mecanismo de vigilancia, constructor de conocimiento, coordinador productivo y formador de cuadros técnicos y políticos, ofrece una estructura de incentivos novedosa que puede afectar cálculos y decisiones políticas nacionales e internacionales, y establecer nuevos pa- 
PiA Riggirozzi

UNASUR: construcción de una diplomacia regional en materia de salud a través de políticas sociales

rámetros de acción colectiva regional. Este es un valor agregado de la política social regional.

\section{Conclusión}

La creciente presencia de las organizaciones regionales en la formulación de las políticas públicas es un tema cada vez mayor en los programas de desarrollo Norte-Sur y Sur-Sur, $\mathrm{y}$ un punto de partida interesante para investigar acerca de los beneficios de la cooperación regional en la provisión de bienes públicos, particularmente aquellos que pueden afectar a ciudadanos en situación de pobreza. Hay un creciente reconocimiento de que las ambiciones e iniciativas de integración regional se extienden más allá de los intercambios comerciales y la inversión, reconociendo la validez y sinergias de los organismos regionales y la ampliación de los derechos (a la salud, pero no solo estos). Pero poco se sabe sobre cuáles son las intervenciones concretas en este sentido, las potencialidades y los desafíos. El presente artículo contribuye a esta discusión, ofreciendo un análisis sobre la agenda de salud de UNASUR para la región y sus ambiciones globales.

Como se desprende del análisis, más allá de las esferas tradicionales del comercio, finanzas e inversión, a partir de la última década se observan nuevas formas de "diplomacia" regional. El artículo argumenta en favor de una mayor atención a las posibles sinergias entre el regionalismo y la reducción de la pobreza mediante intervenciones específicas. Las dinámicas relacionadas con la salud que se observan en el regionalismo sudamericano podrían considerarse como un paso en esta dirección. Institucionalmente, siguiendo la declaración de la salud como un derecho en la Carta Constitutiva de UNASUR, se ha creado un marco normativo que facilita la estructuración de nuevos mecanismos de apoyo al mejoramiento de las prácticas y la gobernanza en materia de salud a nivel nacional. La creación del ISAGS ha sido fundamental para el avance de estos objetivos, mediante la ejecución de programas y proyectos relacionados con el acceso a medicamentos, la vigilancia epidemiológica, la investigación y el desarrollo aplicado, y la evaluación y desarrollo de capacidades para reformar las prácticas y la institucionalidad. De igual forma, nuevos grupos temáticos regionales y redes de profesionales y las asociaciones médicas nacionales se centran específicamente en las enfermedades "de pobres", como el dengue y el Chagas, y los condicionantes sociales de la salud. Asimismo, se ha prestado apoyo a cuadros políticos y profesionales en la creación de instituciones de las escuelas públicas, la atención primaria y la universalización de la salud en los países de UNASUR. Para fortalecer la autonomía y la capacidad de negociación de sus miembros, UNASUR está trabajando en un catastro de la capacidad industrial para producir medicamentos, la adopción 
de posiciones comunes ante las farmacéuticas y en cuestiones sensibles de derechos y representación en la OMS.

La experiencia de UNASUR Salud da sustancia conceptual a la noción de política social regional definida en relación con la capacidad de los organismos regionales de: (i) crear marcos normativos, capaces de estructurar redes intergubernamentales y de expertos a nivel regional; (ii) actuar como agentes redistributivos en la reasignación de recursos materiales y de conocimiento en apoyo de políticas públicas y ejecución de proyectos, y (iii) facilitar la representación y actuación en bloque en la formulación de demandas y negociaciones en instituciones globales y frente a actores externos.

Por supuesto, futuras coyunturas podrían afectar las bases políticas y sociales del activismo regional en la salud, así como en otras áreas sociales. América Latina tiene una larga historia de aspiraciones regionales truncadas. Sin embargo, en la actualidad es una región en que una diversidad de motivaciones, ideologías y aspiraciones de liderazgo impulsan modelos de integración alternativos. A pesar de las muchas contradicciones y legados, sin duda la región recuperó la capacidad de reafirmar las nuevas modalidades de acción colectiva y diplomática, sustentando la formulación de políticas nacionales y la redistribución transfronteriza. En qué medida esto crea nuevas sinergias para el desarrollo de un genuino regionalismo social, se verá en la capacidad de UNASUR de profundizar la experiencia en salud y traducirla en responsabilidades regionales más amplias, abarcando otros ámbitos del desarrollo social relacionados con el medio ambiente, las migraciones y los derechos de las minorías que, como la salud, son políticamente sensibles. Sin embargo, a diferencia de esta, aún no ocupan un lugar de relevancia en la nueva arquitectura institucional de UNASUR, ni un lugar visible en su acción como bloque. Aun así, lo acontecido con la salud nos indica que aquellos interesados en el regionalismo y el desarrollo social, no pueden ignorar el valor de la región como instancia de decisión política, de negociación y de implementación de metas sociales, dentro y fuera de la región.

\section{BibliogRAFÍA}

Agencia Fiocruz de Noticias (2012). Unasur promotes health systems in South American nations. En http:// isags-unasul.org/noticias_interna. asp lang $=2 \&$ idArea $=2 \&$ idPai $=4387(27$ de septiembre de 2013)

Almeida et al. (2010).R. Eletr. de Com. Inf. Inov. Saúde. Rio de Janeiro, 4(1), p.23-32 Álvarez, M. (2011). Los 20 años del MERCOSUR: una Integración a Dos Velocidades. CEPAL - Serie Comercio internacional $\mathrm{N}^{\circ}$ 108: Santiago de Chile: CEPAL

Ayllón Pino, B. (2012). Contribuciones de Brasil al desarrollo internacional: coaliciones emergentes y cooperación Sur-Sur. Revista CIDOB, 97-98, 189-204

Briceño-Ruiz, J. (2010). From the South American Free Trade Area to the Union of South American Nations: The Trans- 
UNASUR: construcción de una diplomacia regional en materia de salud a través de políticas sociales

formations of a Rising Regional Process. Latin American Policy, 1 (2), 208-29.

Buss, P. y Do Carmo Leal, M. (2009). Global Health and Health Diplomacy. En Cadernos da Saúde Pública, 25 (12).

Caetano, G. (2007). Mercosur: ¿Quo Vadis?. Umbrales de América del Sur, 1(1), 51-64.

CEPAL (2011). UNASUR un Espacio de Desarrollo y Cooperación por Construir. Santiago de Chile: CEPAL.

Deacon, B.; Macovei, M.; Van Langenhove, L.; y Yeates, N. (2010). World-regional social policy and global governance: New research and policy agendas in Africa, Asia, Europe and Latin America. Londres: Routledge.

Di Pietro, L. (2003).La Dimensión Social del Mercosur. Recorrido Institucional y Perspectivas. Presentado en el taller: Integración Regional y la Agenda Social, BID-INTAL, Buenos Aires, 12 y 13 de noviembre de 2003.

Draibe, S. (2007). Social Cohesion and Regional Integration: The MERCOSUR Social Agenda and the Integrationist Social Policy Major Challenges.Cadernos de Saúde Pública, 23 (2), 174-83.

Franco, R. y Di Filippo, A. (1999).Las Dimensiones Sociales de la Integración Regional en América Latina, Santiago de Chile: CEPAL.

Gamble, A. y Payne A. eds. (1996). Regionalism and World Order. Londres: Macmillan.

Grugel, J. (2005). Citizenship and Governance in Mercosur: Arguments for a Social Agenda.Third World Quarterly, 26:7, 1061-1076.

Grugel, J. y Riggirozzi, P. (2012). Post-neoliberalism in Latin America: Rebuilding and Reclaming the State after Crisis. Development and Change, 43(1), 1-21.

Hettne, B. (1999). Globalisation and the New Regionalism: the Second Great Transformation. En Hettne, B., Inotai, A. y Sunkel, O. (eds.).Globalism and the New Regionalism. Londres: Macmillan, 1-24.

Hettne, B. (2008). Regional Actorship and Regional Agency: Comparative Perspectives, presentado en $6^{\text {th }}$ GARNET PhD School 'Global Governance and Regionalism'. Bruselas, 9-13 junio.

Hettne, B. y Söderbaum F. (2000). The rise of regionness. New Political Economy, 5(3), 457-474.

Holveck, J., Ehrenberg, J.,Ault, S.,Rojas, R. y Vasquez, J. (2007).Prevention, control and elimination of neglected diseases in the Americas: Pathways to integrated, inter-programmatic, intersectoral action for health and development.BMC Public Health 7. Disponible en http://www. biomedcentral.com/1471-2458/7/6/. (30 demarzo de 2013).

Hurrell, A. (1995). Regionalism in Theoretical Perspective. En Fawcett, L. y Hurrell, A. (eds).Regionalism in World Politics: Regional Organization and International Order. Oxford: Oxford University Press, 37-73

ISAGS (2013). Report January 2013. Disponible en http://issuu.com/isagsunasur/ docs/informe_ing (13 de marzo de 2013).

Jaime, M., Figueroa, J.P., M. Schmidt, M., Muñoz, S., Kuri-Morales,P. y Silva, J.B. (2012). Epidemiology in Latin America and the Caribbean: current situation and challenges.International Journal of Epidemiology, 41(2): 557-571.

MacDonald, L. y Ruckert, A. (2009).Postneoliberalism in the Americas. Nueva York: Palgrave.

Malamud, A. (2005). Mercosur Turns 15: Between Rising Rhetoric and Declining Achievement.Cambridge Review of International Affairs, 18 (3), 421-436.

Ortiz, I. (2007). Social policy in national development strategies. Policy Note. New York: United Nations Department of Economic and Social Affairs.

Pan American Health Organization (PAHO) (2010). UNASUR's Role in the Vaccination Against Pandemic Influenza. PAHO Immunisation Newsletter, 32(4). Disponible en http://new.paho.org/hq/ dmdocuments/2011/SNE3204.pdf (12 marzo de 2013). 
Perrotta, D y Vázquez, M. (2010). El MERCOSUR de las Políticas Públicas Regionales. Las Agendas en Desarrollo Social y Educación. Centro de Formación para la Integración Regional, Documento de Trabajo 10. Montevideo: Uruguay.

Phillips, N. (2003). The Rise and Fall of Open Regionalism? Comparative reflections on regional governance in the Southern Cone of Latin America.Third World Quarterly, 24 (2), 217-234.

Raso Delgue, J. (2006). Libre Comercio y Políticas Sociales en las Áreas de Integración: la Experiencia del Mercosur.Espacio Abierto, 15 (3), 535-543

Riesco, M. (2010). Binding Material for a Young Giant? Regional Policies in Latin America. En Deacon, B., Macovei, M., Van Langenhove, L., y Yeates, N. (eds.).World-Regional Social Policy and Global Governance: New Research and Policy Agendas in Africa, Asia, Europe and Latin America. London: Routledge, 108-139.

Riggirozzi, P. y Tussie, D. (2012). The rise of post-hegemonic regionalism: The case of Latin America. Netherlands: Springer.

Sanahuja, J. A. (2012). Post-liberal Regionalism in South America: The Case of UNASUR. Robert Schuman Centre for Advance Studies, EUI Working Paper 2012/05.

Scharpf, F. (1996). Negative and Positive Integration in the Political Economy of European Welfare States. En Marks, G. y otros (1996). Governance in the European Union. London: Sage, 15-39.

SELA (Sistema Económico Latinoamericano, 2010). Bulletin 150 on Regional integration inLatin America and the Caribbean. Disponible en http://www.sela. org/attach/258/EDOCS/SRed/2010/07/ T023600004239-0-Boletin_150_MAYO_2010_Ingles__.pdf (13 Маyo 2014)

Soares de Lima, M. y Hirst, M. (2009). Brazil as an Intermediate State and Regional Power: Action, Choice and Responsibilities.International Affairs, 82 (1), 21-40.
Szekely, M. y Birdsall, N. (2003). Bootstraps not Band-Aids: Poverty, Equity and Social Policy in Latin America. Working Paper 24, http://www.cgdev.org/content/ publications/detail/2766. 7 (23 de enero de 2012)

Telò, M. (2007), The European Union and New Regionalism: Regional Actors and Global Governance in a Post-Hegemonic Era. Aldershot: Ashgate.

Tussie, D. (2009). Latin America: Contrasting Motivations for Regional Projects. Review of International Studies, 35 (1), 168-188.

UNASUR (2009).Plan Quinquenal, 20102015. Disponible en http://www.ins.gob. pe/repositorioaps/0/0/jer/rins_documentosunasur/PQ\%20UNASUR\%20Salud. pdf (20 de marzo de 2013).

UNASUR (2009b). Tratado Constitutivo de la Unión de Naciones Sudamericanas. Disponible en http://www.comunidadandina. org/unasur/tratado_constitutivo.htm (3 de marzo de 2014).

UNASUR (2010). Ecuador y República Dominicana subscriben convenio para reconstrucción de Haití. Boletín. Disponible en http://www.pptunasur.com/contenidos. php? $\mathrm{id}=1100 \&$ tipo $=27$ \&idiom $=1$ website (28 de marzo de 2014).

UNASUR Salud (2011).Informe de la Secretaria Pro Tempore. Disponible en http://isags-unasul.org/site/wp-content/ uploads/2011/12/Informe-2011.pdf (28 de marzo de 2014).

UNDP (2011).Regional Integration and Human Development: a Pathway for Africa. New York: UNDP.

Van Langenhove, L. y Warleigh-Lack, A. (2011). Rethinking EU Studies: The Contribution of Comparative Regionalism. Journal of European Integration, 32(6): 451-562.

Walker Echenique (2012). Implementing the IP Chapter of the FTA between Chile and the USA: Criticisms and Realities from a Developing Country Perspective. Disponible enhttp://script-ed.org/? $\mathrm{p}=470$ (30 de marzo de 2013). 